Revista Mexicana de Astronomía y Astrofísica, 55, 211-219 (2019)

DOI: https://doi.org/10.22201/ia.01851101p.2019.55.02.09

\title{
3D HYDRODYNAMIC NUMERICAL MODELS FOR NEBULAE AROUND RUNAWAY WOLF-RAYET STARS
}

\author{
J. Reyes-Iturbide ${ }^{1,2}$, Pablo F. Velázquez ${ }^{2}$, M. Rosado ${ }^{3}$, E. Matías Schneiter ${ }^{4,5}$, and I. Ramírez-Ballinas ${ }^{3}$ \\ Received January 14 2019; accepted May 292019
}

\begin{abstract}
We present 3D hydrodynamical simulations of the circumstellar bubble from a Wolf-Rayet runaway star. In the models two properties were taken into account: (a) the proper motion of the central star through the interstellar medium (ISM) and (b) the evolution of the stellar wind from the red supergiant (RSG) stage to the Wolf-Rayet (WR) stage. From the hydrodynamic results synthetic X-ray maps in the $[0.3-1.2] \mathrm{keV}$ energy range were computed. These maps show that the bubble morphology is affected by the stellar motion, producing a bow shock in the RSG stage that can explain the limb-brightened morphology observed. Additionally, these synthetic maps show filamentary and clumpy appearance produced by instabilities triggered from the interaction between the WR wind and the RSG shell. It was found that these types of collisions can explain the origin of the X-ray emission observed in the nebulae of Wolf- Rayet stars.
\end{abstract}

\section{RESUMEN}

Presentamos simulaciones hidrodinámicas 3D para nebulosas producidas por estrellas Wolf-Rayet con movimiento propio. En los modelos tomamos en consideración el movimiento de la estrella a través del medio interestelar (ISM), la tasa de pérdida de masa y la velocidad del viento estelar correspondientes a las fases de súper-gigante roja (RSG) y Wolf-Rayet (WR). A partir de los resultados numéricos, generamos mapas sintéticos de emisión en rayos $\mathrm{X}$ en el intervalo de energía $[0.3-1.2] \mathrm{keV}$. Los mapas muestran una asimetría debida al choque de proa que se produce en la fase de RSG. Adicionalmente, los mapas presentan estructuras filamentarias originadas en la colisión de las burbujas producidas por la RSG y WR. Esta colisión explica también el origen de la emisión de rayos X que se observa en las nebulosas producidas por estrellas Wolf-Rayet.

Key Words: ISM: bubbles — methods: numerical — stars: evolution — stars: winds, outflows — stars: Wolf-Rayet

\section{INTRODUCTION}

Hot massive stars exhibit fast stellar winds that sweep up the surrounding interstellar medium (ISM) creating structures known as interstellar bubbles. Weaver et al. (1977) described an analytical model used to predict the X-ray emission of such objects,

\footnotetext{
${ }^{1}$ Tecnológico de Estudios Superiores de Tianguistenco, Santiago Tianguistenco, Estado de México, México.

${ }^{2}$ Instituto de Ciencias Nucleares, Universidad Nacional Autónoma de México, Ciudad de México, México.

${ }^{3}$ Instituto de Astronomía, Universidad Nacional Autonóma de México, Ciudad de México, México.

${ }^{4}$ Instituto de Astronomía Teórica y Experimental, Córdoba, Argentina.

${ }^{5}$ Departamento de Materiales y Tecnología, Universidad Nacional de Córdoba, Córdoba, Argentina.
}

considering an inner zone of shock-heated gas with temperatures of order $10^{6} \mathrm{~K}$ enclosed within a dense spherical shell with temperatures of order $10^{4} \mathrm{~K}$. This analytical model considered an isotropic stellar wind with constant mechanical luminosity interacting with a homogeneous ISM, and predicted a centerfilled X-ray sphere surrounded by optical emission.

Wolf-Rayet stars offer an ideal laboratory for the study of such bubbles because they are massive stars (progenitor stellar masses greater than $30 M_{\odot}$ ) with mass loss rates of $\dot{M} \approx 10^{-5}-10^{-6} M_{\odot} \mathrm{yr}^{-1}$ and stellar wind velocities that go from 1000 to $2000 \mathrm{~km} \mathrm{~s}^{-1}$. However, observations show bubbles with asymmetric structures meaning that the predictions of the 
analytical models are not valid in general (Chu et al. 1983; Heckathorn et al. 1982; Miller \& Chu 1993; Marston et al. 1994a,b). This observed asymmetry can be caused either by inhomogeneities in the ISM (Toalá et al. 2017) or by anisotropic mass loss rates (Chita et al. 2007, 2008). An alternative mechanism considers the stellar motion that provides a pressure asymmetry, producing asymmetric stellar wind bubbles. The stellar proper motion is supersonic for many massive stars and produces bow shocks when interacting with the ISM (van Buren \& McCray 1988; Cox et al. 2012; Peri et al. 2012, 2015). Both analytical models (van Buren et al. 1990; Mac Low et al. 1991; van Buren \& Mac Low 1992) and numerical models (Brighenti \& D'Ercole 1995a,b; Mackey et al. 2015; Meyer et al. 2015) have been presented to describe stellar wind bow shocks.

Additionally, filamentary structures and clumps have been observed in these WR nebulae in optical bands (Chu 1981; Chu et al. 1983; Moore et al. 2000; Gruendl et al. 2000; Stock \& Barlow 2010) and X-ray emission (Wrigge et al. 1994, 2005; Chu et al. 2003; Zhekov \& Park 2011; Toalá et al. 2012, 2014). These features can be obtained with numerical models that take into account the evolution of the star (mainly the change in mechanical luminosity over time) and its interaction with the circumstellar gas. The evolution history that precedes the WR phase starts from the main-sequence (MS) phase to either the red supergiant (RSG) or luminous blue variable (LBV) phases. Each stage of the stellar evolution is characterized by its own mechanical luminosity and by the formation of a new shell. The interaction between these shells develops instabilities that give rise to the formation of clumps and filamentary structures that have been reproduced by hydrodynamic simulations (Garcia-Segura et al. 1996a,b; Brighenti \& D'Ercole 1997) and radiation-hydrodynamic simulations (Toalá \& Arthur 2011; Dwarkadas \& Rosenberg 2013; Freyer et al. 2006). An important result obtained from these works is the conclusion that the $\mathrm{X}$-ray luminosity is dominated by the emission from the WR shell rather than the shocked WR wind which is the source of the X-ray in the analytical models (Freyer et al. 2006). The numerical models show that soft X-ray emission can be enhanced by thermal conduction, in agreement with the observations (Toalá \& Arthur 2011).

In this work we tested, by means of threedimensional hydrodynamic numerical simulations, the joint effects of the evolutionary phases of a WolfRayet star that moves through the ISM during its lifetime. The hydrodynamic simulations were carried out with the adaptive grid code YGUAZÚ-A with the aim of reproducing the morphology observed in the X-ray emission. We used the numerical results to obtain synthetic thermal X-ray emission maps. This manuscript is organized in the following way: in $\S 2$ the models of the WR nebulae are presented. In $\S 3$ the initial conditions of the numerical simulations are listed as well as an explanation on how the simulation of the thermal X-ray emission was carried out. The results are given in $\S 4$ and a comparison with observations is given in $\S 5$. Finally in $\S 6$ a summary of the conclusions is presented.

\section{MODELLING WR NEBULA}

The runaway WR nebula was modelled considering the evolution of the central star from RSG to WR. The first step was to simulate the RSG phase which will produce a bow shock as a result of the motion of the runaway star. This was done in the frame of reference of the runaway star, i.e., the star was kept at the center of the computational domain with the ISM moving at the velocity at which the star travels $\left(-V_{\star}\right)$. The momentum balance between the stellar wind ram pressure and the ISM, known as stand-off distance $\left(R_{0}\right)$ (see Wilkin 1996), is:

$$
R_{0}=\left(\frac{\dot{M}_{\mathrm{w}} V_{\mathrm{w}}}{4 \pi n_{0} \mu m_{H} V_{\star}^{2}}\right)^{1 / 2},
$$

where $\dot{M}_{\mathrm{w}}$ and $V_{\mathrm{w}}$ are the stellar mass-loss rate and the wind velocity respectively, $V_{\star}$ is the velocity of the star, $n_{0}$ is the numerical density of the uniform ISM, $\mu$ is the mean atomic mass and $m_{H}$ is the mass of the hydrogen atom. For the RSG stage we chose the values for the mass loss rate and the stellar wind velocity as $\dot{M}_{\mathrm{w}}=\dot{M}_{\mathrm{RSG}}$ and $V_{\mathrm{w}}=V_{\mathrm{RSG}}$ respectively. The cells where the wind is injected were imposed within a sphere of radius $5 \times 10^{17} \mathrm{~cm}$ for both the RSG and the WR stages. At a certain integration time, when the shell reaches $R_{0}$, the RSG stage enters a quasi-stationary state. At this time the second step of the simulation is imposed by replacing the RSG wind with a WR stellar wind.

The parameters for the different winds used in these simulations are summarized in Table 1 and correspond to the values obtained by Garcia-Segura et al. (1996b) for $35 M_{\odot}$ stars. The RSG wind velocities range from $30 \mathrm{~km} \mathrm{~s}^{-1}$ to $75 \mathrm{~km} \mathrm{~s}^{-1}$, with the velocity of the star assumed to be within typical values of $V_{\star}<100 \mathrm{~km} \mathrm{~s}^{-1}$. The table also shows the numerical density values of the ISM. 
TABLE 1

SIMULATED MODELS

\begin{tabular}{cccccccc}
\hline Models & $\begin{array}{c}\dot{M}_{S R G} \\
M_{\odot} \mathrm{yr}^{-1}\end{array}$ & $\begin{array}{c}V_{S R G} \\
\mathrm{~km} \mathrm{~s}^{-1}\end{array}$ & $\begin{array}{c}V_{\star} \\
\mathrm{km} \mathrm{s}^{-1}\end{array}$ & $\begin{array}{c}\dot{M}_{W R} \\
M_{\odot} \mathrm{yr}^{-1}\end{array}$ & $\begin{array}{c}V_{W R} \\
\mathrm{~km} \mathrm{~s}^{-1}\end{array}$ & $\begin{array}{c}n_{0} \\
\mathrm{~cm}^{-3}\end{array}$ & $\begin{array}{c}R_{0} \\
\mathrm{pc}\end{array}$ \\
\hline M1 & $8 \times 10^{-5}$ & 75 & 75 & $3 \times 10^{-5}$ & 2000 & 0.4 & 2.4 \\
M2 & $3 \times 10^{-5}$ & 50 & 75 & $3 \times 10^{-5}$ & 1600 & 0.1 & 2.5 \\
M3 & $8 \times 10^{-5}$ & 50 & 50 & $3 \times 10^{-5}$ & 1600 & 1.0 & 1.9 \\
M4 & $3 \times 10^{-5}$ & 30 & 75 & $3 \times 10^{-5}$ & 1600 & 1.0 & 0.6 \\
\hline
\end{tabular}

The second step in the simulation is carried out until a bubble is formed by the interaction between the WR wind and the shell produced by the RSG stellar wind. We used a constant mechanical luminosity for each stage similar to Garcia-Segura et al. (1996a) and did not include the stellar ionization photon flux. The notation for the models is: model 1 (M1), model 2 (M2), model 3 (M3) and model 4 (M4).

\section{THE NUMERICAL MODEL}

The 3D numerical simulations where carried out with the Yguazú-a hydrodynamical code (Raga et al. 2000, 2002). The code integrates the gas dynamic equations with a second-order accurate implementation of the flux vector splitting method (see van Leer 1982) on a binary adaptive grid, along with a system of rate equation for the atomic/ionic species: [H I], [H II], [He III], [He II], [HeIII], [C II], [C III], [C IV], [N I], [N II], [N III], [O I], [O II], [O III], [O IV], [S II] and $[\mathrm{S} \mathrm{III]}$. A computational domain with a physical size of $(16 \mathrm{pc} \times 8 \mathrm{pc} \times 16 \mathrm{pc})$ along the $x-, y-$, and $z-$ directions, respectively, with a maximum spatial resolution of $9.7 \times 10^{16} \mathrm{~cm}$, was employed. The $x z$-plane was chosen as the plane of the sky.

The numerical simulation provided us with density and temperature distributions which were combined with synthetic X-ray spectra to simulate the X-ray emission of the WR nebulae. The X-ray emissivity coefficient $\left(j_{\nu}(\mathrm{n}, \mathrm{Z}, \mathrm{T})\right)$ was calculated in the limit of low-density regime, which is $j_{\nu}(\mathrm{n}, \mathrm{Z}, \mathrm{T})=\mathrm{n}^{2} \chi(\mathrm{Z}, \mathrm{T})$, where $\chi(Z, T)$ is a function of the metallicity and the temperature. Both the gas electron density (n) and temperature $(T)$ were obtained from the numerical results. The synthetic spectra were obtained with the CHIANTI data base (Dere et al. 1997; Landi et al. 2006), where a thermal optically-thin plasma in ionization equilibrium (IEQ) was considered. To compare with observations the X-ray emission coefficient $\left(j_{\nu}(n, T)\right)$ was calculated in the energy range $[0.3-1.2] \mathrm{keV}$ assuming an ISM absorption due to a hydrogen column density of $N_{H}=3.13 \times 10^{21} \mathrm{~cm}^{-2}$. Abundances in the WR stage were selected from Mesa-Delgado et al. (2014) for the WR nebulae NGC 6888 since this is a WR nebula in which a diffuse X-ray emission was detected (Toalá et al. 2016, 2014; Zhekov \& Park 2011; Wrigge \& Wendker 2002).

\section{RESULTS \\ 4.1. RSG Stage}

As mentioned in $\S 2$, the RSG phase was simulated until an integration time corresponding to when the stand-off distance $R_{0}$ was reached in each model. The top row panels of Figures 1, 2, 3, and 4 (left panel) display the temporal evolution of the density distribution on the $x z$ - plane. These show a characteristic bow shock bubble produced by the interaction of the isotropic RSG wind and the planeparallel wind (modelled as the ISM moving towards the star due to our reference system moving with the star).

The axisymmetry of the bow shock bubble is broken by the development of Vishniac instabilities (Vishniac 1983). At this stage of the evolution the RSG wind is dense enough to produce a radiative terminal shock forming a thin, dense shell which is observed in the [H II] density distribution for M1 (see Figure 5 top row panel). We show that this region has temperatures, at $80 \mathrm{kyr}$, of $\approx 10^{4} \mathrm{~K}$ as shown in Figure 6. The models are hydrodynamic and do not take into account the stellar radiation, so they are expected to produce a thin shell in the H II density distribution, produced by the shock wave. In works where stellar radiation is included the ionization regions are extended and fully ionized (Dwarkadas \& Rosenberg 2013; Meyer et al. 2015).

At $80 \mathrm{kyr}$ and $100 \mathrm{kyr}$ for models M1 and M2, respectively, and at $120 \mathrm{kyr}$ for both models M3 and M4 the balance between the ram pressure of the stellar wind and the ISM are achieved. Stellar evolution 


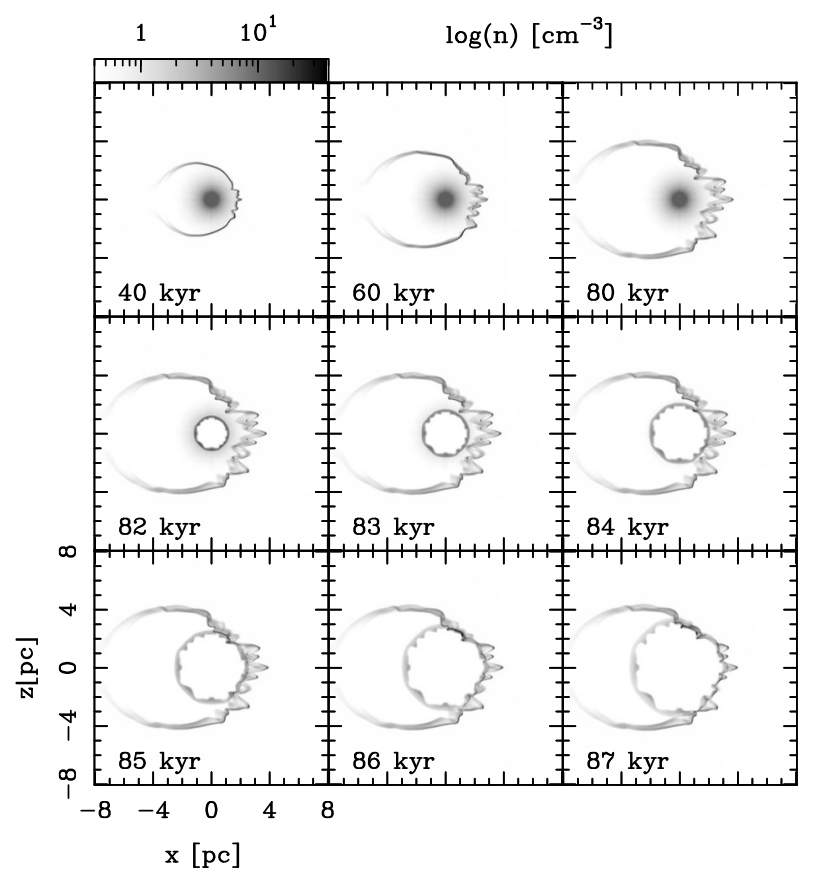

Fig. 1. For the M1 run temporal evolution of the density distribution is shown, on the $x z$-plane, for several integration times. The three top panels display the interaction between the RSG stellar wind and the plane-parallel ISM wind, which produces a characteristic bow shaped morphology. The evolution of the WR stellar wind into the asymmetric RSG shell is shown in the middle three panels, while the three bottom panels display the interaction between the RSG and WR shells. Both axes are given in units of pc, while the logarithmic grey scale gives the density in units of $\mathrm{cm}^{-3}$.

models for $35 M_{\odot}$ predict that the duration of this stage is $\approx 200$ kyr (Garcia-Segura et al. 1996b), approximately twice to what was obtained.

\subsection{WR Stage}

As mentioned above the WR stage starts at times $t=80, t=100 \mathrm{kyr}$ for $\mathrm{M} 1$ and $\mathrm{M} 2$, respectively, and at $t=120 \mathrm{kyr}$ for both M3 and M4, i.e. when the WR stellar wind is turned on. The middle row panels in Figures 1, 2, 3, and 4 (middle panel) show how the WR stellar wind evolves through the RSG wind material. A spherical shell is formed due to the fastslow wind interaction which takes place inside the smooth RSG medium, showing the development of Rayleigh-Taylor instabilities. A similar behavior is observed for the ionized hydrogen, H II density distribution in Figure 5 (middle panel) for M1. At integration times of $t=84,103,124$ and $122 \mathrm{kyr}$ for M1, M2, M3, and M4, respectively, the collisions between RSG and WR shells have just started.

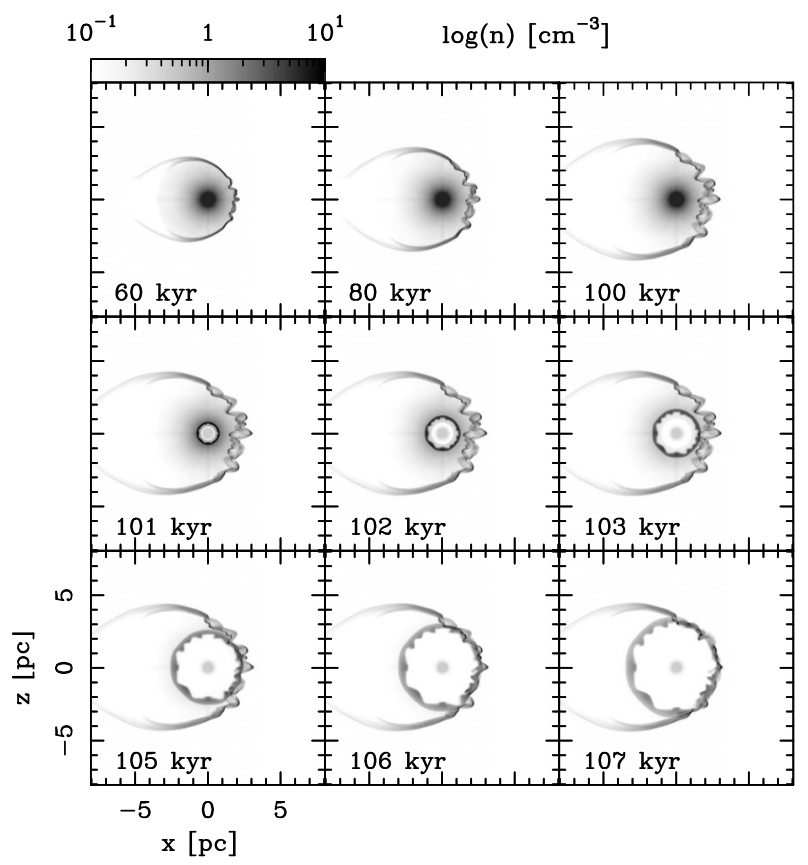

Fig. 2. The same as in Figure 1 but for M2.

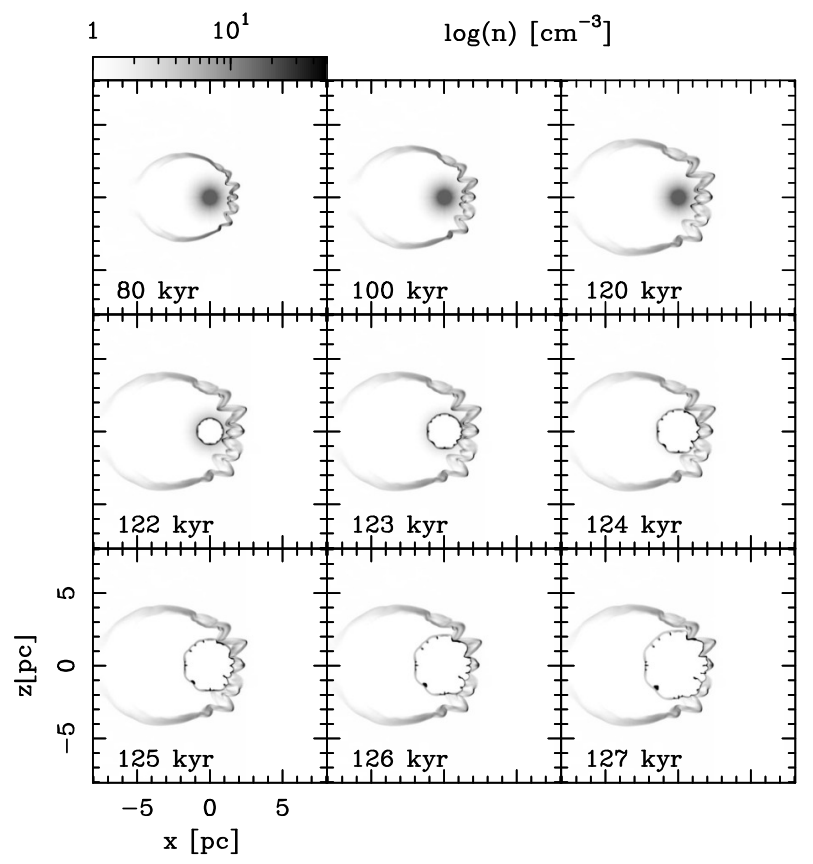

Fig. 3. The same as in Figure 1 but for M3.

\subsection{Shell Collision}

The hypothesis is that Wolf-Rayet nebulae are originated in the collision between the shells formed during the WR and RSG stages. In order to check this, the evolution of the collision of the wind shells was followed. The panels in the bottom row of Figures $1,2,3$, and 4 (middle and right panel) display 


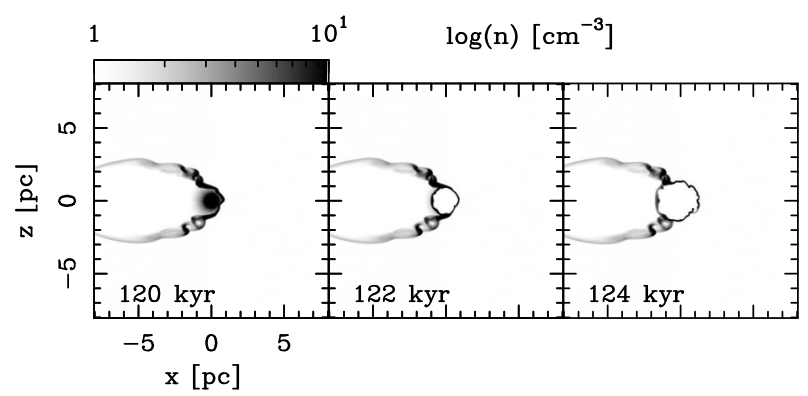

Fig. 4. The same as in Figure 1 but for M4.

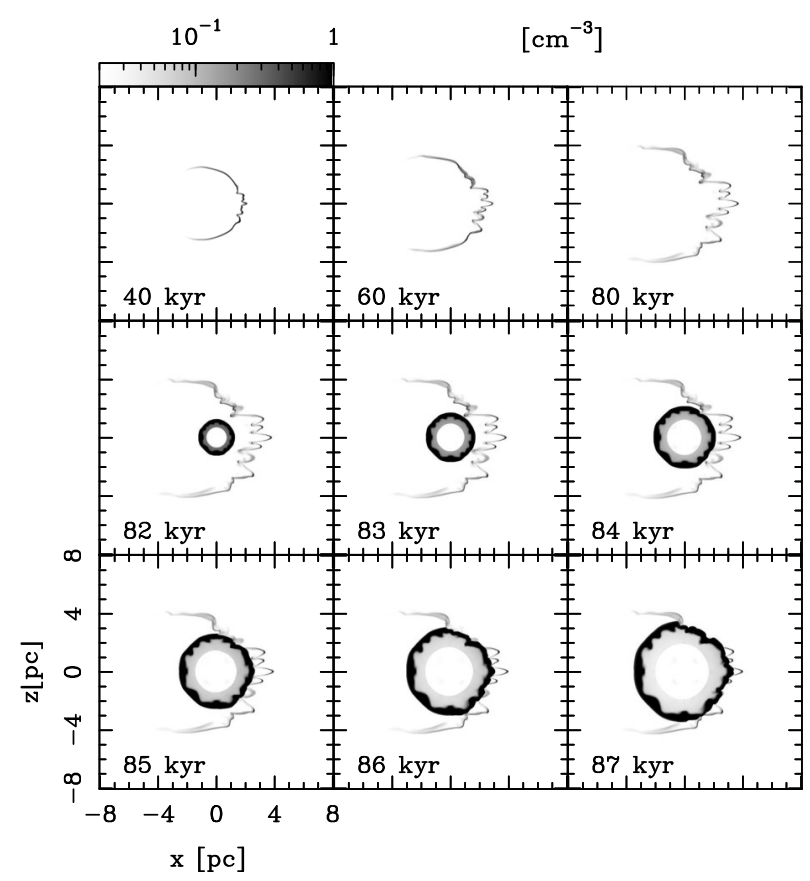

Fig. 5. For the M1 run, the temporal evolution of the number density distribution of the ionized hydrogen, [H II], is shown. These synthetic maps were computed considering that the line of the sight is along the $y$ - axis (i.e. $x z$-plane is the plane of the sky). Both axes are in units of pc. The logarithmic gray scale is given in units of $\mathrm{cm}^{-3}$

this evolution. They show that for M1 the start of the interaction occurs at an integration time between 84 and $85 \mathrm{kyr}$. The end of this interaction occurs at a time of $87 \mathrm{kyr}$, when the WR wind has passed completely through the bow shock structure generated by the RSG wind. Similarly, for M2 the shell collision takes place at an integration time [105-107] kyr, for M3 [124 - 127] kyr, and $122 \mathrm{kyr}$ for M4.

\subsection{X-ray Emisson From Shell Collision}

Synthetic maps were created for each model for $\mathrm{X}$-ray emission in the $[0.3-0.7],[0.7-1.2]$, and

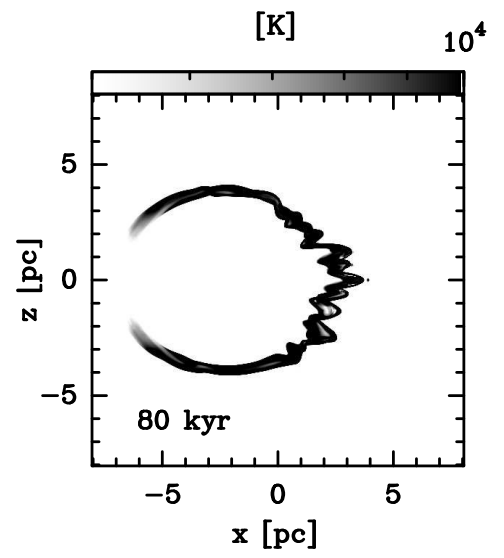

Fig. 6. For M1 the synthetic temperature map at precollision time $t=80 \mathrm{kyr}$ is shown. This synthetic map was computed considering that the line of the sight is along the $y-$ axis (i.e. $x z$ - plane is the plane of the sky). Both axes are in units of pc. The logarithmic gray scale gives the temperature in units of degrees $\mathrm{K}$.

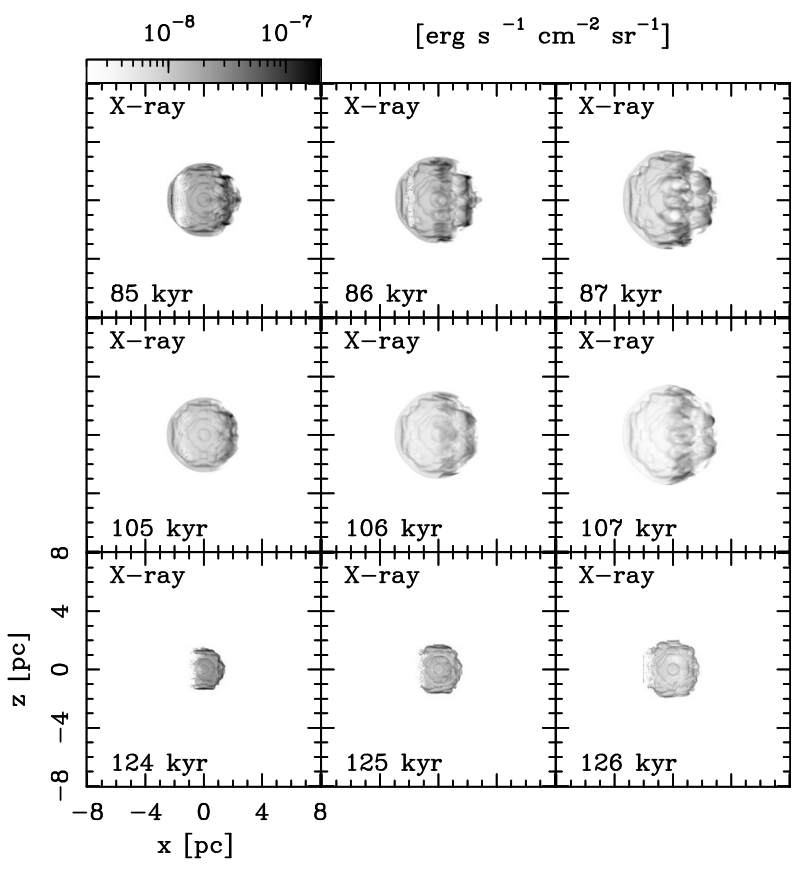

Fig. 7. X-ray emission in the $[0.3-1.2] \mathrm{keV}$ band. Top row panel, middle row panel, and bottom row panel for M1, M2, and M3, respectively. Both axes are in units of pc. The logarithmic gray scale gives the flux in units of erg s $\mathrm{s}^{-1} \mathrm{~cm}^{-2} \mathrm{sr}^{-1}$

$[1.2-3] \mathrm{keV}$ bands. We found only soft emission, so there is no considerable emission in the interval $[1.2-3.0] \mathrm{keV}$. The emission is significant for the $([0.3-1.2]) \mathrm{keV}$ energy band which is displayed in the top, middle, and bottom row panels of Figure 7, for 

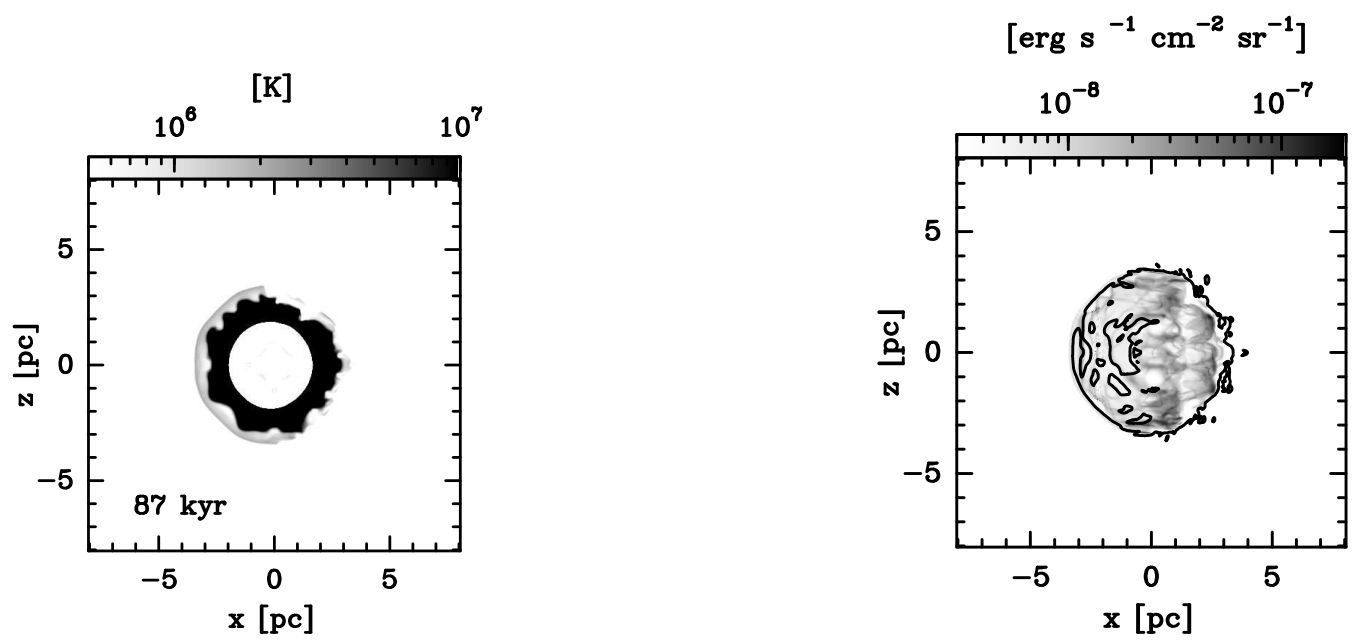

Fig. 8. Left panel. For M1 the synthetic temperature map at collision time $t=87$ kyr is shown. This synthetic map was calculated considering that the line of the sight is along the $y$ - axis (i.e. $x z-$ plane is the plane of the sky). Both axes are in units of pc. The logarithmic gray scale gives the temperature in units of degrees K. Right panel. For Mod1 a comparison between the X-ray (in gray scale) and the $[\mathrm{H} \alpha]$ emissions is shown (black contours at level $4 \times 10^{-9}$ ) for an integration time of $87 \mathrm{kyr}$. Both axes are in pc. The logarithmic gray scale is in units of $\mathrm{erg} \mathrm{s}^{-1} \mathrm{~cm}^{-2} \mathrm{sr}^{-1}$

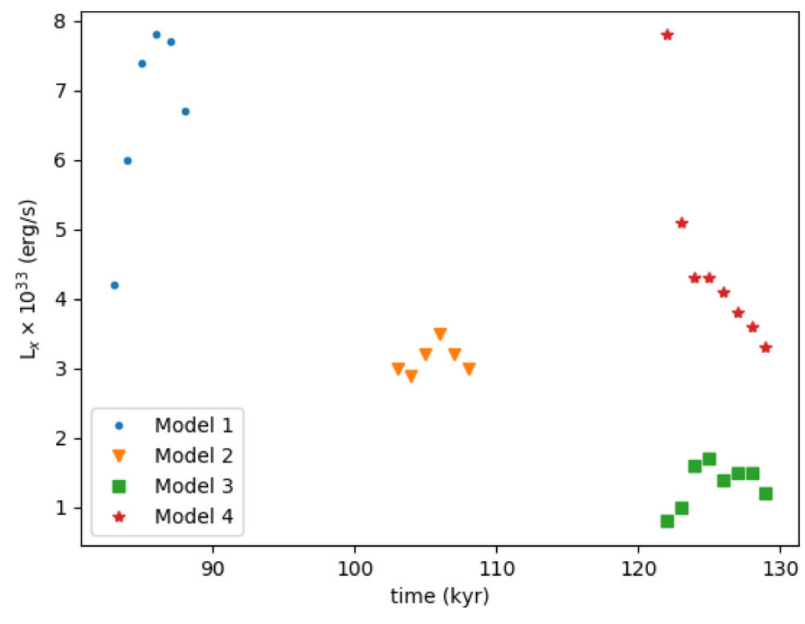

Fig. 9. X-ray luminosity in the $[0.3-1.2] \mathrm{Kev}$ band as a function of time for M1, M2, M3, and M4. The color figure can be viewed online.

models M1, M2, and M3, respectively (these maps correspond to the collision times). These maps show a clumpy and filamentary morphology. The size of the emitting region increases as the collision evolves in time, to a lesser extent for M3. This suggests that this nebula could be the product of the collision between the WR and the RSG winds. Figure 8 (left panel) shows the spatial distribution of the temperature at a time $t=87 \mathrm{kyr}$ for M1. A shell with temperatures $10^{6}-10^{7} \mathrm{~K}$, surrounded by an outer shell with temperatures of $10^{5}-10^{4} \mathrm{~K}$ is observed.
In order to analyze the spatial distribution of the $\mathrm{H} \alpha$ and X-ray emission, the right panel of Figure 8 compares both emissions. In gray scale the thermal $\mathrm{X}$-ray emission is displayed while the $\mathrm{H} \alpha$ emission is shown as black contours. From this figure we can see that the optical emission appears to wrap around the thermal X-ray emission. An important result is that the maximum in X-ray emission occurs in the collision, as shown in Figure 9. The maximum emission for M1 takes place at a time of $86 \mathrm{kyr}$ with a value of $7.8 \times 10^{33} \mathrm{erg} \mathrm{s}^{-1}$. For M2 it occurs at $107 \mathrm{kyr}$ with a value of $3.5 \times 10^{33} \mathrm{erg} \mathrm{s}^{-1}$, M3 reaches maximum at $125 \mathrm{kyr}$ with value $1.7 \times 10^{33} \mathrm{erg} \mathrm{s}^{-1}$, and for M4 the maximum occurs at $122 \mathrm{kyr}$, with a value of $7.8 \times 10^{33} \mathrm{erg} \mathrm{s}^{-1}$.

\section{DISCUSSION}

The standard model from Weaver et al. (1977) proposes thermal conduction at the interface between hot gas (or shocked wind) and cold and dense gas (or shocked interstellar medium) with temperatures of $10^{6} \mathrm{~K}$ and $10^{4} \mathrm{~K}$, respectively, as the mechanism for explaining the X-ray emission in stellar wind bubbles. The density of the hot gas increases due to the photoevaporation caused by the thermal conduction in the interface, producing an increase on the X-ray emission. However, their predictions are not compatible with the low luminosities observed.

Soft X-ray emission has been detected in WolfRayet nebulae with luminosities in the range $L_{x}=10^{33}-10^{34} \mathrm{ergs}^{-1}$ (Chu et al. 2003; Toalá et 
TABLE 2

LUMINOSITIES OF THE MODELS

\begin{tabular}{|c|c|c|c|c|c|}
\hline Model & {$[0.3-0.7]^{\mathrm{a}} \mathrm{keV}$} & $\begin{array}{c}L_{x}[\mathrm{erg} / \mathrm{s}] \\
{[0.3-1.2]^{\mathrm{a}} \mathrm{keV}}\end{array}$ & {$[0.3-0.7]^{\mathrm{b}} \mathrm{keV}$} & {$[0.3-1.2]^{\mathrm{b}} \mathrm{keV}$} & Time $[\mathrm{kyr}$ \\
\hline M1 & $6.9 \times 10^{33}$ & $7.8 \times 10^{33}$ & $1.6 \times 10^{31}$ & $6.5 \times 10^{31}$ & 86 \\
\hline M2 & $3.0 \times 10^{33}$ & $3.5 \times 10^{33}$ & $0.8 \times 10^{31}$ & $3.5 \times 10^{31}$ & 107 \\
\hline M3 & $1.5 \times 10^{33}$ & $1.7 \times 10^{33}$ & $0.2 \times 10^{31}$ & $2.2 \times 10^{31}$ & 125 \\
\hline M4 & $6.8 \times 10^{33}$ & $7.8 \times 10^{33}$ & $1.5 \times 10^{31}$ & $9.9 \times 10^{31}$ & 122 \\
\hline
\end{tabular}

${ }^{\mathrm{a}}$ Corrected for absorption.

${ }^{\mathrm{b}}$ With absorption.

al. 2012, 2015, 2016). Our models show that the total X-ray luminosity $L_{x}$ (corrected by absorption) at the time of collision is close to these values, summarized in Table 2. A large part of the emission in the $[0.3-1.2] \mathrm{keV}$ band comes from the subinterval $[0.3-0.7] \mathrm{keV}$; for our models it represents $\approx 90 \%$. The soft X-ray emission is associated to a short period of time of the order of $\approx 1$ to $2 \mathrm{kyr}$ (see Figure 9). Hydrodynamic models with thermal conduction show a similar behavior, although soft X-ray emission lasts for periods of time $<50 \mathrm{kyr}$ (Toalá \& Arthur 2011). If we consider the ISM absorption in our calculation of the X-ray luminosities they turn out to be lower by two orders of magnitude (see Table 2).

The synthetic maps show a clumpy structure formed by instabilities due to the wind interaction. We also note that the brightness distribution of these emissions exhibits limb-brightening (at most one order of magnitude higher to the right of our synthetic nebulae). The limb-brightening is observed to a lesser degree for M3 (see Figure 7).

The synthetic X-ray emission maps (see Figure 7) show that most of the emission is coming from several filaments produced by the wind collisions between the shells from the RSG and WR stages, while according to the theory the X-ray profile should be centrally filled. Previous works have shown that soft $\mathrm{X}$-ray emissions in models of WR nebulae come from clumps and can be enhanced by thermal conduction (Freyer et al. 2006; Toalá \& Arthur 2011). In spite of our models being hydrodynamic, which do not consider the effect of thermal conduction, we measure luminosities similar to the ones obtained by Freyer et al. (2006) and Toalá \& Arthur (2011).

Only 12 runaway WR stars in our Galaxy have been reported. For WR 3, WR 148, and WR 124 corrected absorbed luminosities of $\approx L_{x}=10^{32} \mathrm{erg} \mathrm{s}^{-1}$
(Rosslowe \& Crowther 2015) were obtained, one order of magnitude lower than the ones obtained in these models, see Table 2. The asymmetric nebula observed in NGC 6888 can be due to the motion of the star through the ISM. The star WR 136 is classified as a runaway star with a velocity (from the proper motion) $\approx 100 \mathrm{~km} / \mathrm{sec}$. However, the orientation between the bow shock symmetry axis and the velocity is 90 degrees (van Buren et al. 1995).

\section{CONCLUSIONS}

We have carried out 3D hydrodynamical simulations in order to model the evolution and emission of WR nebulae. As in previous works (GarciaSegura et al. 1996a) we have followed the evolution of the stellar wind of the central star from the RSG to WR phase. We considered the velocity of the central star in the nebula as moving in an ISM with a constant density. This produces a RSG shell with bow shock morphology. From the density and temperature distributions obtained from our numerical results several synthetic X-ray emission maps (in the $[0.3-1.2] \mathrm{keV}$ energy range) were generated. These synthetic X-ray emission maps show a non-uniform brightness distribution. Limb-brightened, clumpy, and filamentary emissions are displayed in Figure 7, in good agreement with observational results (Gruendl et al. 2000; Moore et al. 2000). This spatial distribution of the emission is generated by the joint action of the stellar motion and the stellar evolution from the RSG to WR phases. The limb-brightened structure is produced by the collision of the SRG shell with the WR stellar wind. This collision produces an enhancement of the density and the temperature, giving as a result an increase in the X-ray emission, with a difference between the low and high emission regions of one order of magnitude at most. The total X-ray luminosity computed from the sim- 
ulations displays an increase after the shell collision (see Figure 9). The emission in hard X-rays is weak, only the soft X-ray emission is considerable.

In summary, our model, which includes the stellar wind evolution from the RSG to the WR stage and the motion of the central star of the WR nebulae, can reproduce an asymmetric morphology in X-ray emission.

We thank the anonymous referee for her/his very useful comments and suggestions, which helped us to improve the previous version of this manuscript. We also thank Artemi Camps-Fariña for reading this manuscript. Finally, we Acknowledge finantial support from TNM-SEP, PAPIIT IG100218, CONACYT CY-253085 and UNAM DGAPA-PAPIIT IN 109919.

\section{REFERENCES}

Brighenti, F. \& D'Ercole, A. 1995, MNRAS, 273, 443 1995, MNRAS, 277, 53

1997, MNRAS, 285, 387

Chita, S. M., van Marle, A. J., Langer, N., \& GarcíaSegura, G. 2007, RMxAC, 30, 80

Chita, S. M., Langer, N., van Marle, A. J., García-Segura, G., \& Heger, A. 2008, A\&A, 488, L37

Chu, Y.-H. 1981, ApJ, 249, 195

Chu, Y.-H., Treffers, R. R., \& Kwitter, K. B. 1983, ApJS, 53, 937

Chu, Y.-H., Guerrero, M. A., Gruendl, R. A., GarcíaSegura, G., \& Wendker, H. J. 2003, ApJ, 599, 1189

Cox, N. L. J., Kerschbaum, F., van Marle, A.-J., et al. 2012, A\&A, 537, A35

Dere, K. P., Landi, E., Mason, H. E., Monsignori Fossi, B. C., \& Young, P. R. 1997, A\&AS, 125, 149

Dwarkadas, V. V. \& Rosenberg, D. L. 2013, HEDP, 9, 226

Freyer, T., Hensler, G., \& Yorke, H. W. 2006, ApJ, 638, 262

Garcia-Segura, G., Langer, N., \& Mac Low, M.-M. 1996, A\&A, 316, 133

Garcia-Segura, G., Mac Low, M.-M., \& Langer, N. 1996, A\&A, 305, 229

Gruendl, R. A., Chu, Y.-H., Dunne, B. C., \& Points, S. D. 2000, AJ, 120, 2670

Heckathorn, J. N., Bruhweiler, F. C., \& Gull, T. R. 1982, ApJ, 252, 230

Landi, E., Del Zanna, G., Young, P. R., et al. 2006, ApJS, 162,261

Mackey, J., Gvaramadze, V. V., Mohamed, S., \& Langer, N. 2015, A\&A, 573, A10
Mac Low, M.-M., van Buren, D., Wood, D. O. S., \& Churchwell, E. 1991, ApJ, 369, 395

Marston, A. P., Chu, Y.-H., \& Garcia-Segura, G. 1994, ApJS, 93, 229

Marston, A. P., Yocum, D. R., Garcia-Segura, G., \& Chu, Y.-H. 1994, ApJS, 95, 151

Mesa-Delgado, A., Esteban, C., García-Rojas, J., et al. 2014, ApJ, 785, 100

Meyer, D. M.-A., Langer, N., Mackey, J., Velázquez, P. F., \& Gusdorf, A. 2015, MNRAS, 450, 3080

Miller, G. J. \& Chu, Y.-H. 1993, ApJS, 85, 137

Moore, B. D., Hester, J. J., \& Scowen, P. A. 2000, AJ, 119,2991

Peri, C. S., Benaglia, P., Brookes, D. P., Stevens, I. R., \& Isequilla, N. L. 2012, A\&A, 538, A108

Peri, C. S., Benaglia, P., \& Isequilla, N. L. 2015, A\&A, $578, \mathrm{~A} 45$

Raga, A. C., Navarro-González, R. \& Villagrán-Muniz, M. 2000, RMxAA, 36, 67

Raga, A. C., de Gouveia Dal Pino, E. M., NoriegaCrespo, A., Mininni, P. D., \& Velázquez, P. F. 2002, A\&A, 392, 267

Rosslowe, C. K. \& Crowther, P. A. 2015, MNRAS, 447, 2322

Stock, D. J. \& Barlow, M. J.2010, MNRAS, 409, 1429

Toalá, J. A. \& Arthur, S. J. 2011, ApJ, 737, 100

Toalá, J. A., Guerrero, M. A., Chu, Y.-H., et al. 2012, ApJ, 755, 77

Toalá, J. A., Guerrero, M. A., Gruendl, R. A., \& Chu, Y.-H. 2014, AJ, 147, 30

Toalá, J. A., Guerrero, M. A., Chu, Y.-H., \& Gruendl, R. A. 2015, MNRAS, 446, 1083

Toalá, J. A., Marston, A. P., Guerrero, M. A., Chu, Y.H., \& Gruendl, R. A. 2017, ApJ, 846, 76

Toalá, J. A., Guerrero, M. A., Chu, Y.-H., et al. 2016, MNRAS, 456, 4305

van Buren, D. \& McCray, R. 1988, ApJ, 329, L93

van Buren, D., Mac Low, M.-M., Wood, D. O. S., \& Churchwell, E. 1990, ApJ, 353, 570

van Buren, D. \& Mac Low, M.-M. 1992, ApJ, 394, 534

van Buren, D., Noriega-Crespo, A., \& Dgani, R. 1995, AJ, 110, 2914

van Leer, B. 1982, International Conference on Numerical Methods in Fluid Dynamics, 8th ed. (Berlin: Springer), 507

Vishniac, E. T. 1983, ApJ, 274, 152

Weaver, R., McCray, R., Castor, J., Shapiro, P., \& Moore, R. 1977, ApJ, 218, 377

Wilkin, F. P. 1996, ApJ, 459, L31

Wrigge, M., Wendker, H. J., \& Wisotzki, L. 1994, A\&A, 286,219

Wrigge, M. \& Wendker, H. J. 2002, A\&A, 391, 287

Wrigge, M., Chu, Y.-H., Magnier, E. A., \& Wendker, H. J. 2005, ApJ, 633, 248

Zhekov, S. A. \& Park, S. 2011, ApJ, 728, 135 
E. Matías Schneiter: Instituto de Astronomía Teórica y Experimental CP: X5000BGR, Córdoba, Argentina.

E. Matías Schneiter: Departamento de Materiales y Tecnología, Universidad Nacional de Córdoba CP: X5016GCA, Córdoba, Argentina.

J. Reyes-Iturbide: Tecnológico de Estudios Superiores de Tianguistenco, Carretera Tenango-La Marquesa Km. 22, Santiago Tianguistenco, Estado de México, México (jr.iturbide@gmail.com).

J. Reyes-Iturbide and Pablo F. Velázquez: Instituto de Ciencias Nucleares, Universidad Nacional Autónoma de México, Apdo. Postal 70-543, CP: 04510, Ciudad de México, México (jr.iturbide@gmail.com).

I. Ramírez-Ballinas and M. Rosado: Instituto de Astronomía, Universidad Nacional Autonóma de México, Apdo. Postal 70-264, C.P. 04510, Ciudad de México, México. 